\title{
DETECÇÃO DE REGIÕES SUSPEITAS DE LESÃO NA MAMA EM IMAGENS TÉRMI- CAS UTILIZANDO SPATIOGRAM E REDES NEURAIS
}

\author{
SUSPECT DETECTION OF REGIONS OF INJURY IN BREAST IN THERMAL IM-AGES \\ USING SPATIOGRAM AND NEURAL NETWORKS
}

\section{DETECCIÓN DE ZONAS SOSPECHOSAS DE LESIÓN EN LA MAMA EN IMÁGENES TÉR- MICAS UTILIZANDO SPATIOGRAM Y REDES NEURALES}

\author{
Paulo Sérgio Aguiar Junior \\ Caio Nogueira Silva Belfort \\ Aristófanes Corrêa Silva \\ Pedro Henrique Bandeira Diniz \\ Rita de Cassia Fernandes de Lima \\ Aura Conci \\ Anselmo Cardoso de Paiva
}

\begin{abstract}
Resumo: Este trabalho propõe uma metodologia para identificar regiões suspeitas de lesão baseada nas assime-trias da mama esquerda e direita de imagens de termogramas. O estudo é pautado em imagens de pacientes do Hospital Universitário da Universidade Federal de Pernambuco (UFPE), capturadas por câmera infravermelha. Inicialmente as imagens são manualmente segmentadas. Em seguida, os seios são registrados usando a transformação B-spline. Além disso, como o corpo humano tem uma simetria radial das temperaturas, uma lesão, eventualmente, leva uma assimetria destas regiões, em seguida, o spatiogram é usado para identificar essas regiões assimétricas. Finalmente, apenas as regiões com temperaturas superiores à média são mantidas, com base no fato de que o câncer tem a temperatura mais elevada do que o restante mama. Após esse processo são extraídas características (Variação dos pixels, a média, o desvio padrão, o índice de Geary e Dimensão Fractal de Higuchi) para a classificação dessas regiões restantes em lesão ou não lesão utilizando-se uma rede neural artificial com perceptron em multicamadas. A metodologia apresentou $75 \%$ das regiões classificadas corretamente, indicando que o spatiogram e a média das temperaturas das regiões assimétricas são métodos bem promissores para identificação de regiões suspeitas de conter lesão.
\end{abstract}

Palavras-chave: Termografia. Câncer. Spatiogram. Mama. Rede-neural.

Abstract: This paper proposes a methodology to identify suspicious regions of injury based on asymmetries of left and right breasts of thermograms images.. The study is based on images captured by infrared camera from patients at the University Hospital of the Federal University of Pernambuco. Initially the images are manually segmented. Then, the sinuses are recorded using the B-spline transformation. Furthermore, as the human body has a radial symmetry of temperatures, damage eventually leads asymmetry of these regions, then the spatiogram is used to identify those asymmetric.regions. Finally, only the regions with higher than average temperatures are maintained, based on the fact that the cancer has a higher temperature than the rest of the breast. After this process features are extracted (Variation of pixels, the mean, standard deviation, index Geary and Higuchi Fractal Dimension) for the classification of regions remaining in injury or no injury using an artificial neural network Multilayer perceptron. The methodology showed $75 \%$ of correctly classified regions, indicating that the spatiogram and the average temperatures of the asymmetric regions are well promises methods to identify regions suspected of containing lesion.

Keywords: Thermography. Cancer. Spatiogram. Breast. Neural-network.

Resumen: En este trabajo se propone una metodología para identificar las regiones sospechosas de lesión basado en las asimetrías de la mama izquierda y derecha de las imágenes termogramas. El estudio se basa en las imágenes capturadas por la cámara infrarroja de los pacientes en el Hospital Universitario de la Universidade Federal de Pernambuco. Inicialmente, las imágenes son segmentadas manualmente. Luego, los senos se registran utilizando la transformación B-spline. Además, como el cuerpo humano tiene una simetría radial de temperaturas, daños eventualmente conducen a una asimetría de estas regiones, entonces el spatiogram se utiliza para identificar las regiones asimétricas. Finalmente, basado en el hecho de que el cáncer tiene una temperatura más alta que el resto de la mama, sólo las regiones con temperaturas más alta que la temperatura media son mantenidas. Después de este proceso se extraen características (Variación de píxeles, la media, desviación estándar, Dimensión índice y Higuchi Geary fractal) para la clasificación de las regiones restantes en lesiones o ninguna lesión utilizando un perceptrón multicapa red neural artificial. La metodología mostró $75 \%$ regiones clasificados correctamente, lo que indica que las temperaturas spatiogram y media de las regiones son métodos asimétricos bien promete para identificar regiones sospechosas de contener lesión.

Palabras clave: Termografía. Cáncer. Spatiogram. Mama. Redes neuronales.

\footnotetext{
*Artigo recebido maio 2013
}

Aprovado em junho 2013 


\section{INTRODUÇÃO}

O câncer de mama tem sido há décadas o tipo mais comum entre as mulheres. Apesar da elevada taxa de incidência, a mortalidade causada por ele tem diminuído desde o final dos anos oitenta, graças ao avanço das pesquisas em métodos para o diagnóstico precoce. A mamografia, método de detecção mais amplamente empregado, não é tão eficaz para mulheres com mamas densas, cirurgicamente alteradas ou com menos de 40 anos. Além disso, há preocupação em relação ao risco de radiação ionizante e a queixas dos pacientes sobre desconforto devido à compressão dos seios (KOAY; HERRY; FRIZE, 2004).

$\mathrm{Na}$ procura por outras técnicas de imagens para complementar mamografia, a termografia (ou termograma) apresentou-se como um ótimo método para melhorar a eficiência na detecção do câncer de mama (GAUTHERIE; GROS, 1980). No entanto, a sua utilização prematura e a introdução mal controlada no diagnóstico de câncer de mama trouxe muitas críticas negativas por gerar muitos falsos positivos. Nos últimos tempos, com a melhoria das câmeras infravermelhas em face da alta aplicação na indústria, a investigação na área voltou com maior força, pois equipamentos mais modernos levaram a melhores resultados. Com isso, pesquisadores demonstraram que o termograma tem um grande potencial para a detecção precoce e prognóstico do câncer, usado em conjunto com a mamografia (FRIZE; HERRY; ROBERGE, 2002). Como o calor metabólico é realizado em todo o corpo, principalmente por convecção através do fluxo de sangue, um aumento na temperatura cutânea geralmente indica um aumento no fluxo sanguíneo em torno do "ponto quente" (LEEK, 2001). A hipertermia em torno do tumor é causada por dois fatores: a maior taxa metabólica das células cancerosas e a angiogênese do tumor. A angiogênese (formação de novos vasos sanguíneos) do tumor tem sido bastante investigada por ser um significativo indicador de prognóstico do câncer de mama (CHU; HUANG; CHANG, 1998).

O corpo humano possui certa simetria em relação ao eixo vertical, com isso uma pessoa saudável possui a temperatura de uma região da mama próxima à temperatura da outra. O objetivo desde trabalho é identificar regiões suspeitas de lesão baseado nas assimetrias da mama esquerda e direita de imagens de termogramas. Para realizar este objetivo usamos o histograma espacial (ou spatiogram) para identificar regiões que possuam as temperaturas com certo grau de assimetria, características e rede neural para classificá-las em lesão ou não lesão.

O trabalho está dividido da seguinte forma. Na seção 2 é mostrado o estado da arte, na seção 3 é exposta a fundamentação teórica para o trabalho. E na seção 4 é descrita a utilização do spatiogram, características e redes neurais. Na seção 5 são mostrados os resultados estatísticos dos casos utilizados no trabalho e duas imagens são mostradas com os resultado dos passos da metodologia. Por fim, na seção 6 é exposta a visão sobre os resultados obtidos neste estudo.

\section{TRABALHOS RELACIONADOS}

Este trabalho tem como base diversos trabalhos publicados anteriormente em âmbito internacional e nacional. Foram utilizadas técnicas de trabalhos que estão relacionados com o prognóstico do câncer. Gautherie e Gros (1980) mostraram que a termografia indicava ser uma boa ferramenta para o prognóstico, mas até então pouco explorada. Com Leek (2001) podemos compreender o padrão formado pela angiogênese do câncer, assim ressaltando que as regiões do câncer da mama são as que apresentam maior temperatura comparada ao restante da mama. Frize, Herry e Roberge (2002) compararam regiões da mama para identificar as regiões suspeitas por setores. Um método que se mostrou bem promissor para tal finalidade foi o spatiogram, que é descrito por Birchfield e Rangarajan (2005). Tal método é interessante, pois não são considerados apenas os valores dos pixel, mas também sua posições, com isso regiões que possuem pixel quentes concentrados em um local serão identificadas de modo diferente de uma região com a mesma quantidade de pixel quentes mas não concentrados. Resmini (2011) mostra que os índices descritores de texturas são ótimos para o trabalho com imagens térmicas. Koay, Herry e Frize (2004) mostraram a utilização de rede neural e o ótimo desempenho da rede neural com perceptron em multicamadas.

\section{CONCEITOS BÁSICOS}

\subsection{Registro de imagem}

Apesar de o corpo humano possuir simetria radial, geralmente as mamas não possuem a mesma forma e os seus centros não estão posicionados na mesma linha vertical. Para calcular a região assimétrica, é necessário que as mamas possuam a mesma forma e estejam na mesma direção. Assim sendo, existe a necessidade de registrar as duas mamas, para que haja a correção em relação à rotação e a deformação. Para realizar o registro foi utilizada transformação por deformação $B$-spline, largamente usada para resolver problemas de registro deformável (HAJNAL; HILL; HAWKES, 2001).

\subsection{Spatiogram}

O histograma $\left(h_{A}(z)\right)$, de uma imagem em níveis de cinza $A$ constitui uma operação global a qual indica a frequência de ocorrência desses 
níveis ou do brilho $z$ da imagem. Desse modo o histograma de uma imagem que possua $L$ níveis de cinza é representado por um vetor de $L$ elementos (FALCÃO, 2008).

O histograma dá uma ideia global da dinâmica de uma cena e tem aplicações em inúmeras transformações de imagens. O mesmo pode ser associado a diferentes imagens e sua informação é invariante a operações de rotação e translação da imagem. Mesmo assim não são adequados para a detecção de objetos porque não armazenam informações a respeito da distribuição espacial dos pixels. Para algumas imagens pertencentes e não pertencentes à classe de interesse, seus histogramas podem ser muito similares, o que os faz insuficientes para a detecção de objetos. O spatiogram mantém todas essas informações espaciais de cada bin(cada elemento $p=(x, y)$ pertencente ao domínio da imagem).

Para calcular o spatiogram de uma imagem de $N$ pixels, onde a contagem do bin é representada por $n_{b}$ e os bins por $b$, aplica-se a Equação 1:

$$
\text { (1) } n_{b}=\sum_{i=1}^{N} \delta_{i b}
$$

Outras informações necessárias para calcular o spatiogram são a média e a covariância. A média é calculada a partir da posição espacial dos pixels que pertence a cada bin, descrito pela Equação 2. Onde $\mu_{h}$ é média das posições espaciais para cada bin:

(2) $\mu_{b}=\frac{1}{\sum_{j=1}^{N} \delta_{i b}} \sum_{i=1}^{N} x_{i} \delta_{i b}$

E a covariância é calculada a partir de cada $\operatorname{bin}\left(\Sigma_{b}\right)$, descrito pela Equação 3:

(3) $\sum_{b}=\frac{1}{\sum_{j=1}^{N} \delta_{j b}} \sum_{i=1}^{N}\left(x_{i}-\mu_{b}\right)\left(x_{i}-\mu_{b}\right)^{T} \delta_{i b}$

O spatiogram é composto por essas três componentes, que vão conter as informações espaciais daquela imagem em questão.

Neste trabalho, o spatiogram é utilizado para calcular o nível de assimetria de regiões das duas mamas, de forma que um valor alto deste índice de assimetria significa que essa região é suspeita de conter uma lesão.

Para calcular a diferença entre as imagens da mama esquerda e direita, são usadas janelas $(A e B)$ que percorrem as imagens, calculando o spatiogram para os valores dos pixels dessas janelas. A comparação entre os dois spatiogram é calculada pela Equação 4, onde $k$ representa a quantidade de bins na imagem, e $\beta$ é o índice que indica o nível de assimetria das regiões da mama:
(4) $\beta=\frac{1}{\mathrm{k}}|| \sum_{j=1}^{N} \Sigma_{b_{A}}|-| \sum_{j=1}^{N} \Sigma_{b_{A}}||$

Os valores de $\beta$ variam de 0 até 1 , onde 0 significa pouco diferente e 1 muito diferente. Como as mamas não apresentam simetria perfeita há a necessidade de traçarmos um limiar para identificar a partir de qual valor do índice $\beta$ as regiões podem ser consideradas assimétricas. O limiar adotado em nossos experimentos foi 0,35 .

Mais informações sobre o spatiogram podem ser obtidas em Birchfield, Rangarajan (2005).

\subsection{Rede Neural Artificial (RNA)}

As redes neurais artificiais se fundamentam nos estudos sobre a estrutura do cérebro humano para tentar emular sua forma inteligente de processar informações. Apesar de desconhecermos a maneira pela qual o cérebro manipula informações complexas (BEALE; JACKSON,1990), sabemos que a modelagem do conhecimento contido em um problema específico pode ser representada através de interconexões entre células nervosas. Estruturalmente, a rede neural artificial, também conhecida por modelo conexionista de computação, assemelha-se à rede neural biológica pela composição de seus neurônios e pela conexão entre eles (WASSERMAN, 1989).

O processo de treinamento pode ser visto como um problema de otimização, onde se quer minimizar o erro quadrático médio de todo o conjunto de dados de treinamento. Este problema pode ser resolvido de duas maneiras diferentes: variando o padrão de otimização heurística por emparelhamento simulado ou através de técnicas de otimização mais específicas, como o algoritmo genético no algoritmo do gradiente de descida especializada, como o de retro propagação (brackpropagtion).

O algoritmo mais usado é de retropropagação, mas ele tem algumas limitações relativas, como a extensão de ajustamento para os pesos em cada interação. Este problema foi resolvido usando um algoritmo mais avançado como Rprop.

O Rprop (resilient back-propagation) é um algoritmo de adaptação global que realiza treinamento supervisionado em lote em redes do tipo Perceptron Multicamada (MLP-Multilayer perceptron) (RIEDMILLER, 1994). Ele procura eliminar a influência negativa do valor da derivada parcial na definição do ajuste dos pesos. Essa influência negativa ocorre porque, quando a saída de um neurônio for próximo de 0 (ou 1 ) e a saída desejada for 1 (ou 0), a derivada será próxima de 0 , fazendo com que os pesos destes neurônios recebam um ajuste mínimo, próximo de 0 . 
O algoritimo Rprop elimina esse problema utilizando apenas o sinal da derivada, e não o seu valor. O sinal indica a direção do ajuste dos pesos (aumentar ou diminuir o peso anterior). O tamanho do ajuste dos pesos é dado por um "valor de atualização".

O valor de atualização é definido por um processo de adaptação que depende do sinal derivada do erro com relação ao peso a ser ajustado.

Segundo a regra de adaptação utilizada pelo Rprop, quando a derivada parcial do erro em relação a um peso mantém o seu sinal, o que indica que seu último ajuste reduziu o erro cometido, o valor de atualização é aumentado, acelerando a convergência do treinamento. Quando a derivada parcial muda de sinal, o que indica que o último ajuste foi grande demais, o valor de atualização é reduzido, mudando a direção dos ajustes (BRAGA; CARVALHO; LUDERMIR, 2007, p.63).

\section{4 Índice de Geary}

Índice de Geary é uma medida de autocorrelação espacial. A autocorrelação espacial expressa a correlação de observação adjacente do mesmo fenômeno. Trata-se de três dimensões espaciais. Contudo, a correlação pode também ser expressa em proximidade temporal.
O índice de Geary é definido na Equação 5:

$$
C=\frac{(N-1) \sum_{i} \sum_{j} \mathrm{w}_{i j}\left(x_{i}-x_{j}\right)^{2}}{2 w \sum_{i}\left(x_{i}-\bar{x}\right)^{2}}
$$

Onde $N$ é o número de unidades espaciais indexadas por $i$ e $j$. $X$ é variável de interesse, $X$ é a média de $X, w_{i j}$ é uma matriz de pesos espaciais, e $w$ é a somá do todos (RESMINI, 2011).

\subsection{Dimensão Fractal de Higuchi}

Dimensão Fractal de Higuchi é uma medida muito adequada à representação de geometrias mais irregulares. A dimensão fractal pode ser avaliada por diversas maneiras aproximadas e diversos algoritmos de computação. Elá é utilizada em sinais unidimensionais para medir a complexidade do sinal segundo o conceito fractal. Este conceito descreve melhor a geometria encontrada na natureza, sendo esta geometria não trivial de ser representada sinteticamente. O coenficiente de Higuchi é definido na Equação 6 (abaixo), onde $k$ é o degrau aplicado ao cálculo, $N$ é a quantidade de dados da sequência $x, m$ é o início da sequência para um determinado $\mathrm{k}$ variando de 0 até $k-1$, $L_{m}$ é a soma das diferenças do dado atual com o dado $k$ posições anterior e $\frac{\frac{(N-1)}{[N-m} k}{k} k$ é a normalização da acumulação (RESMINI, 2011).

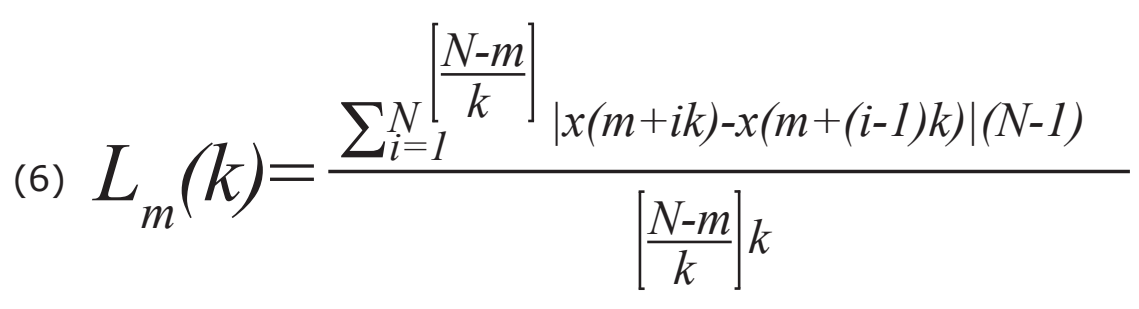

\section{METODOLOGIA PROPOSTA}

A metodologia proposta segue os passos descritos na figura 1.

Para fazer a análise das imagens, é necessária primeiro a extração das regiões de interesse nas imagens (mamas esquerda e direita). Essas regiões foram extraídas manualmente, com o especialista selecionando as bordas das mamas por meio do mouse. 0 resultado desta operação pode ser visto na Figura 2.

Figura 1 - Fluxograma da metodologia

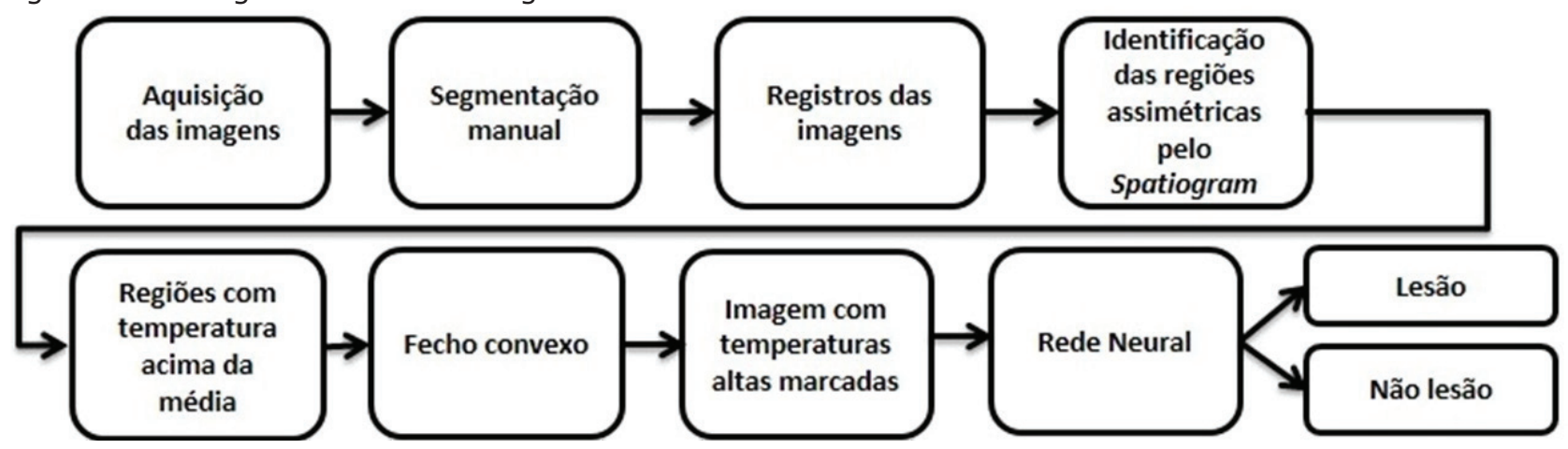

Fonte: Elaborado pelos autores 
Figura 2 - Imagem segmentada manualmente

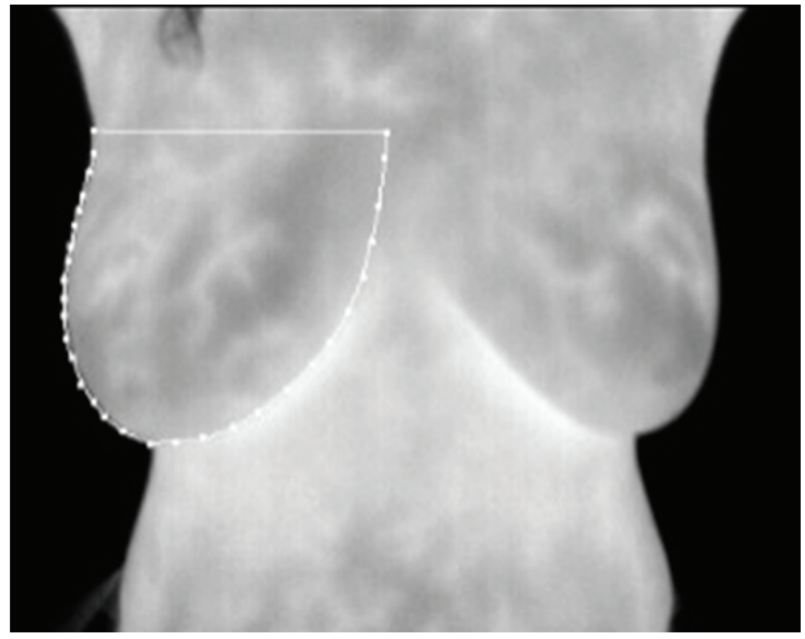

Fonte: Adaptação de Proeng (2012)

Depois de extraídas as imagens das mamas, é necessário que elas tenham a forma mais semelhante possível para que a verificação de regiões assimétricas seja efetuada corretamente. Desta forma, realiza-se o registro das mamas com o algoritmo B-spline.

Uma das imagens será a mama fixa e a outra será a imagem a ser registrada (ou imagem móvel). A imagem a ser registrada terá sua forma alterada para se sobrepor à imagem fixa, obtendo o menor erro possível em relação à forma da mama, ou seja, as duas imagens possuirão a forma semelhante. A figura 3 apresenta o resultado da aplicação do registro, onde (a) é a mama direita, (b) é a mama esquerda espelhada e (c) a mama esquerda registrada.

Depois de registradas as imagens das mamas, é usado o spatiogram para identificar as regiões que possuem temperaturas diferentes nas duas imagens, isto é, assimétricas. As áreas em verde nas imagens da figura 4 ilustram as regiões assimétricas nas duas mamas:

Figura 4 - Regiões assimetricas. (a) mama esquerda e (b) mama direita
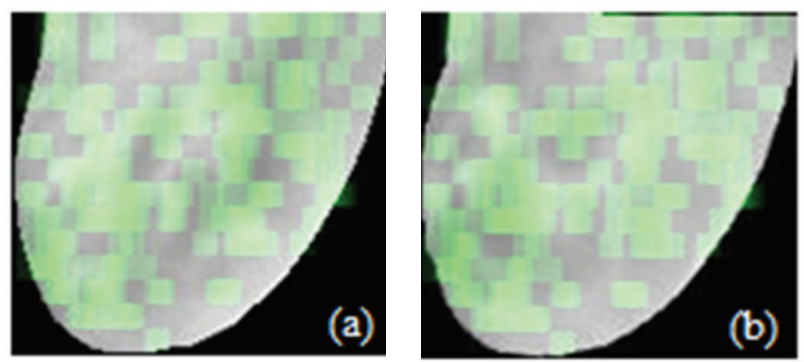

Fonte: Elaborado pelos autores

Em seguida, a partir das regiões identificadas pelo spatiogram como assimétricas, é feita uma classificação para eliminar as regiões de baixas temperaturas e restar apenas as regiões de temperaturas acima de um limiar. Esse limiar é a média das temperaturas.
Figura 3 - Resultado do registro da imagem
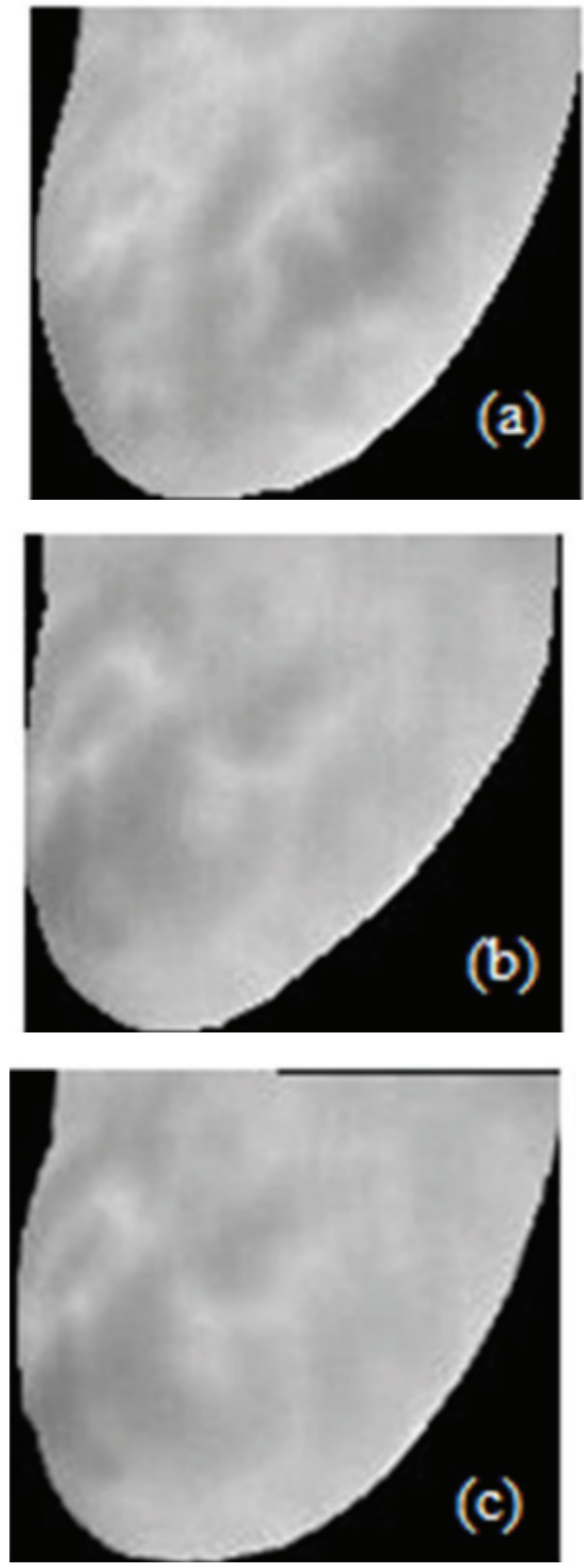

Fonte: Elaborado pelos autores

Para classificar essas regiões tomamos como base a média de todas as médias das temperaturas das regiões das duas mamas identificadas pelo spatiogram. A Equação 7 mostra como é calculada a média de cada região. é a média de uma região e $M x N$ o tamanho dessa região:

$$
\text { (7) } \tau_{i}=\frac{1}{M x N} \sum M_{j=1}^{M} \sum N_{i=1} \chi(i, j)
$$

Na Equação 8, é calculada a média das médias das regiões individuais $\left(\tau_{t}\right)$ e $z$ é o número de regiões identificadas pelos spatiogram. 


\section{(8) $\tau_{t}={ }_{z}^{1 \sum_{i=1}^{z}} \tau_{i}$}

Com base no $\tau_{t}$, são agrupadas as regiões que apresentam altas temperaturas e depois é usado um fecho convexo para melhorar a identificação. A Figura 5 ilustra este processo:

Figura 5 - Mamas com regiões com temperaturas altas marcadas por fecho convexo
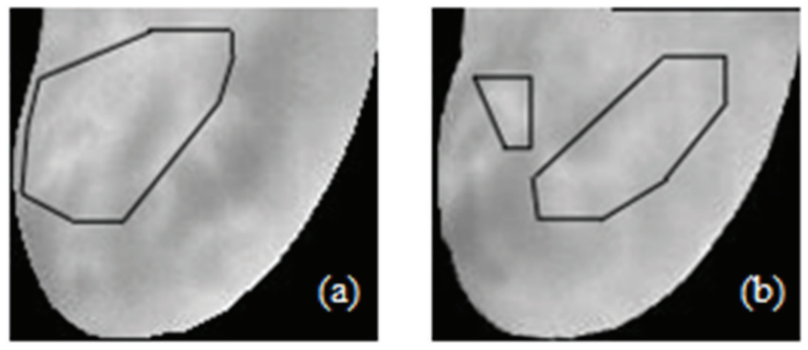

Fonte: Elaborado pelos autores

Com base nas regiões com temperaturas altas marcadas por fecho convexo, utiliza-se a rede neural para classificar essas regiões e indicar aquelas que realmente possam conter uma lesão.

Para criar o vetor de característica para a MLP com algoritmo de treinamento de retro propagação com Rprop foram utilizados cinco índices amplamente conhecidos. São eles: a variação dos pixels, a média, o desvio padrão, o índice de Geary e Dimensão Fractal de Higuchi.

Foi extraído um vetor de características para análise de cada região, a partir do qual essas regiões são analisadas e classificadas em dois casos: lesão ou não lesão.

\section{RESULTADOS}

Este trabalho utiliza imagens provenientes da base de dados do projeto PROENG (PROENG, 2012). As imagens foram adquiridas de pacientes do Hospital Universitário da Universidade Federal de Pernambuco (UFPE). As imagens das pacientes foram adquiridas utilizando uma câmera térmica modelo Flir ThermaCAM S45 (FLIR SYSTEM, 2004), com a resolução de $320 \times 240$ pixels. O banco de imagens possui 320 imagens de mamas saudáveis e com lesão. Deste total apenas 10 imagens de mamas que possuíam lesão tiveram o diagnóstico concluído a partir de biópsia ou exames estruturais como a mamografia. Neste trabalho será utilizada uma amostra de imagens de 20 pacientes que tiveram o diagnóstico concluído a partir de biópsia ou exames estruturais como a mamografia. Desta amostra utilizada, 10 pacientes apresentam ambas as mamas saudáveis e 10 pacientes apresentam pelo menos uma mama com patologia.

Em todas as imagens analisadas com o spatiogram e com as médias são detectadas entre 1 e 3 regiões suspeitas de possuir lesão. Em todas as imagens de mama que foram diagnosticadas pelo especialista com lesão, a mesma ficou localizada em uma dessas regiões assimétricas.

A MLP apresenta na camada de entrada 50 neurônios, com uma camada oculta com 24 neurônios e a camada de saída contém 1 neurônio. A camada de saída e a oculta possuem como função de ativação a sigmoide simétrica. A MLP apresenta fator de incremento do Rprop de 1,2 e fator de decremento de 0,5 e um delta variando de 0 a 50 . O momento de aprendizagem é 0 .

Com a utilização da rede neural para classificar as regiões, teve-se $75 \%$ de regiões classificadas corretamente, dos $25 \%$ das regiões classificadas de modo incorreto, $16 \%$ foram de falsos positivos e $9 \%$ de falsos negativos.

A seguir será exemplificada a aplicação da metodologia proposta em dois casos com diferentes diagnósticos.

A Figura 6 apresenta um termograma com um carcinoma ductal invasivo indicado por um quadrado marcado pelo especialista. Na figura 7 tem-se o resultado da metodologia proposta, em que na mama direita (a) foi identificada a mesma área da lesão descrita na Figura 6. Na figura 8 , temos a eliminação das regiões que não pertencem à lesão com a rede neural.

Figura 6 - Termograma com carcinoma ductal invasivo

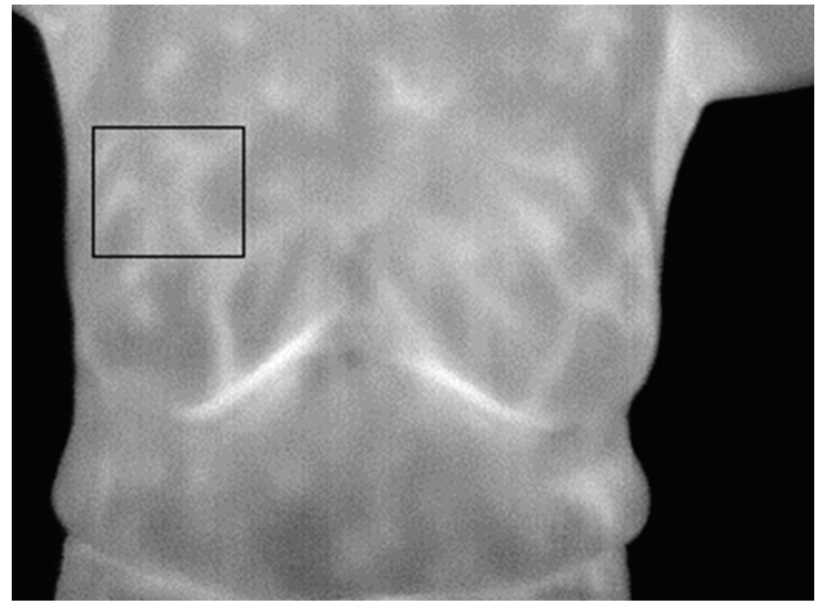

Fonte: Adaptação de Proeng (2012)

Figura 7 - Regiões classificadas como suspeitas de ser lesão
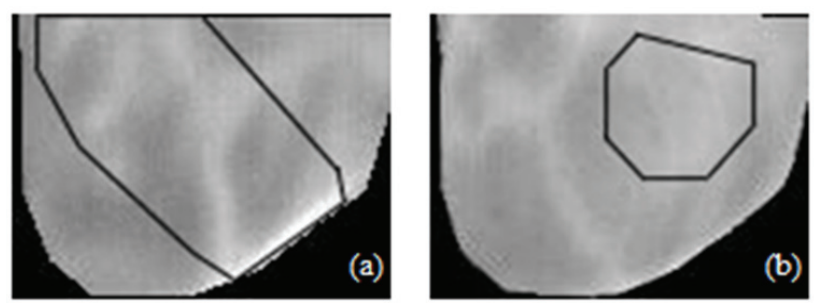

Fonte: Elaborado pelos autores

A figura 9 apresenta um termograma com um abscesso indicado por um quadrado. $\mathrm{Na}$ Figura 10, tem-se o resultado da metodologia proposta, na qual na mama esquerda (a) foi identificada a mesma área da lesão descrita na figura 9. Na figura 11 temos a eliminação 
das regiões que não pertencem à lesão com a rede neural.

Figura 8 - Regiões classificadas pela rede neural que possivelmente tenha lesão

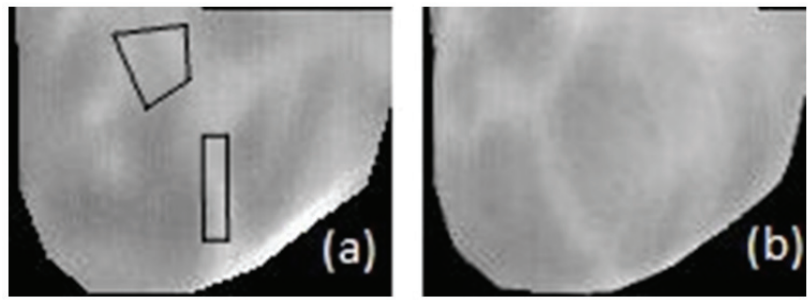

Fonte: Adaptação de Proeng (2012)

Figura 9 - Termograma com abscesso

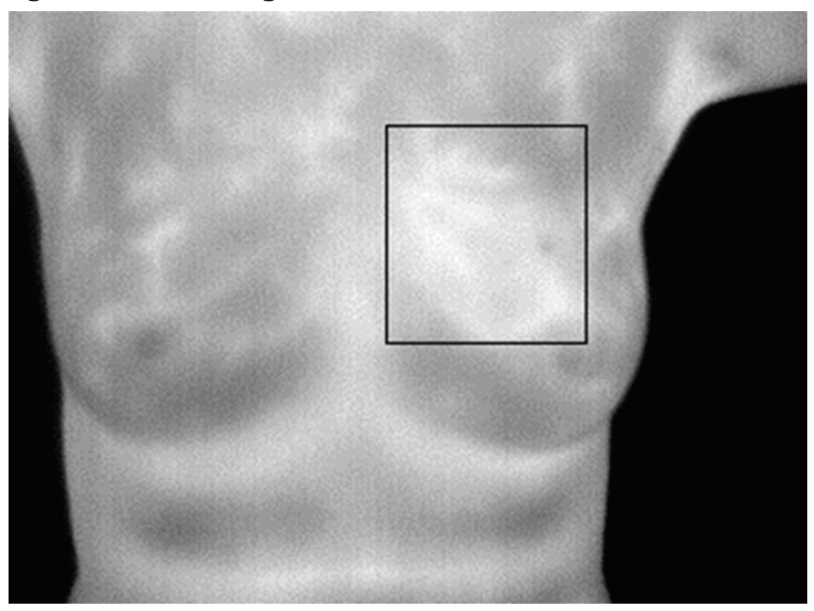

Fonte: Adaptação de Proeng (2012)

Figura 10 - Regiões classificadas como suspeitas de ser lesão
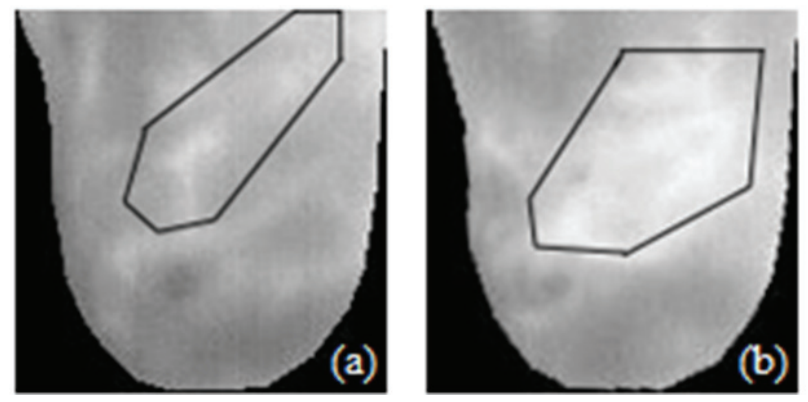

Fonte: Elaborado pelos autores

Figura 11 - Regiões classificadas pela rede neural que possivelmente tenha lesão
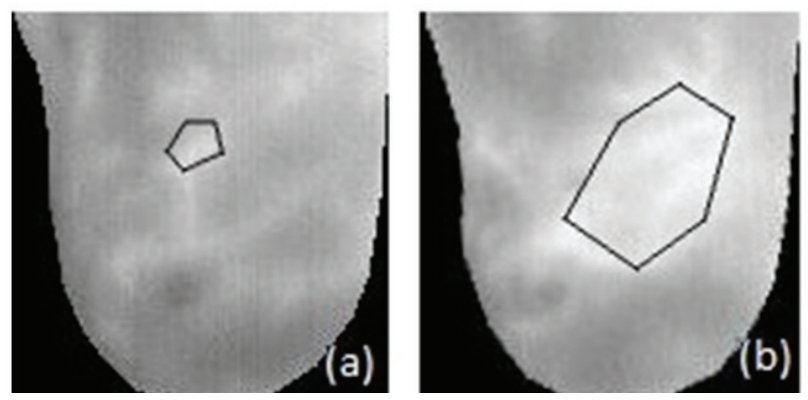

Fonte: Elaborado pelos autores

\section{CONSIDERAÇÕES FINAIS}

Neste trabalho foi desenvolvida uma metodologia para detectar regiões suspeitas de conter lesão e classificá-las utilizando rede neural. Foi verificado que o spatiogram junto com a média das temperaturas das regiões assimétricas e a média, desvio padrão, índice de Geary e Dimensão Fractal de Higuchi na MLP produziram resultados bem promissores para identificação de regiões suspeitas de conter lesão, pois conseguimos identificar bem as regiões com pouca ou muita diferença de temperatura, principalmente pela detecção das lesões específicas na base de imagens, o que contribui para os bons resultados alcançados na classificação da rede neural, em que $75 \%$ de classificação correta foram obtidos.

Os resultados adquiridos com a rede neural foram bons, levando em conta a pequena base de imagens disponíveis no momento. Com o aumento da base de imagens os resultados tendem a melhorar a adição de outros métodos para o pré-processamento da imagem tende a melhorar o resultado final, diminuindo os falsos positivos e falsos negativos.

\section{AGRADECIMENTOS}

Agradecemos a Fapema e CNPq pelo apoio financeiro e ao NCA pela estrutura fornecida.

\section{REFERÊNCIAS}

BEALE, R; JACKSON, T. Neural computing: an introduction. Bristol: Adam Hilger, 1990

BIRCHFIELD, S. T.; RANGARAJAN, E S.

Spatiograms versus histograms for regionbased tracking. In: IEEE COMPUTER SOCIETY CONFERENCE ON COMPUTER VISION AND PATTERN RECOGNITION, 2005. San Diego: IEE Computer Society, 2005. p. 1158-1163.

BRAGA, A. P.; CARVALHO A. P. L. F.; LUDERMIR, E. R. B. Redes neurais artificiais. 2.ed. Rio de Janeiro: LTC, 2007.

CHU, J. S.; HUANG, C. S.; CHANG, K. J. The prognostic significance of tumor angiogenesis in Taiwanese patients with invasive ductal breast carcinomas. Cancer letters, v.134, n.1, p. 7-14, 1998.

FLIR SYSTEMS. ThermaCAM TM S45 Infrared Camera: user manual, 2004. Disponível em: <http://yellotec.com/pdf/S45_Datasheet. pdf $>$. Acesso em: 19 jun. $201 \overline{2}$.

FRIZE, M.; HERRY, C.; ROBERGE, R.

Processing of thermal images to detect breast cancer: a comparison. In: JOINT IEEE EMBS/ BMES CONF., 2., 2002, Houston Houston, TX, 2002. p. $1159-1160$.

GAUTHERIE M.; GROS, C. M. Breast thermography and cancer risk prediction. Cancer, v. 45, p. 51-6, 1980. 
HAJNAL, J. V.; HILL, D. L.; HAWKES, D. J. Medical image registration. Boca Raton, FL: , CRC Press, 2001.

KOAY, J.; HERRY, C.; FRIZE, M.

Analysis of breast thermography with an artificial neural network. In: ANNUAL INTERNATIONAL CONFERENCE OF THE IEEE EMBS, 26., 2004. p.1-5, 2004.

LEEK, R. D. The prognostic role of angiogenesis in breast cancer. Anticancer research, v. 21, n. 6B, p. 4325-4331, 2001.

PROENG. Image processing and image analyses applied to mastology, 2012. Disponível em: <http://visual.ic.uff.br/ en/proeng/>. Acesso em: 19 jun. 2012.
RESMINI, R. Análise de imagens térmicas da mama usando descritores de textura. Rio de Janeiro: Universidade Federal Fluminense, 2011.

RIEDMILLER, M. Rprop - Description and implementation details: technical report. Karlsruhe: University of Karlsruhe, 1994.

WASSERMAN, P. D. Neural computing: theory and practice. New York: Van Nostrand Rei

WASSERMAN, P. D. Neural computing: theory and practice. New York: Van Nostrand Reinhold, 1989. 